\title{
Processing Homonyms in the Kana-to-Kanji Conversion
}

\author{
Masahito Takahashi \\ Fukuoka University \\ 8-19-1, Nanakuma, \\ Jonan-ku, Fukuoka, \\ 814-01, Japan \\ takahasi@helio.tl. \\ fukuoka-11.ac.jp
}

\author{
Tsuyoshi Shinchu \\ Fukuoka University \\ 8-19-1, Nanakuma, \\ Jonan-ku, Fuknoka, \\ 814-01, Japan \\ shinchu@helio.tl. \\ fukuoka-u.ac:.jp
}

\author{
Kenji Yoshimura \\ Fukuoka University \\ 8-19-1, Nanakuma, \\ Jonan-ku, Fukuoka, \\ 814-01, Japan \\ yosimuractlsun.tl. \\ fukuoka-11.ac.jp
}

\author{
Kosho Shudo \\ Fukuoka University \\ 8-19-1, Nanakuma, \\ Jonan-ku, Fukuoka, \\ 814-01, Japan \\ shudo@tlsun.tl. \\ fukuoka-u.ac.jp
}

\begin{abstract}
This paper proposes two new methods to identify the correct meaning of Japanese homonyms in text based on the noun-verb co-occurrence in a sentence which can be obtained easily from corpora. The first method uses the nour co-occurrence data sets, which are constructed from the above co-oceurrence relation, to select the most feasible word among homonyms in the scope of a sentence. The second uses the far cooccurrence data sets, which are constructed dynamically from the near cooccurrence data sets in the course of processing input sentences, to select the most feasible word among homonyms in the scope of a sequence of sentences. An experiment of kana-to-kanji(phonogranto-ideograph) conversion has shown that the conversion is carried out at the accuracy rate of $79.6 \%$ per word by the first method. This accuracy rate of our method is $7.4 \%$ higher than that of the ordinary method based on the word occurrence frequency.
\end{abstract}

\section{Introduction}

Processing homonyms, i.e. identifying the correct meaning of homonyms in text, is one of the most important phases of $k a n a-$ to-kanji conversion, currently the most popular method for iuputting Japanese characters into a computer. Recently, several new methods for processing homonyms, based on neural networks(Kobayashi, 1992) or the co-occurrence relation of words(Yamamoto,1992) , have been proposed. These methods apply to the co-occurrence relation of words not only in a sentence but also in a sequence of sentences. It appears impracticable to prepare a neural network for co-occurrence data large enough to handle 50,000 to 100,000 Japanese words.

In this paper, we propose two new methods for processing Japanese homonyms based on the co- occurrence relation between a noun and a verb in a sentence. We have defined two co-occurrence data sets. One is a set of nouns accompanied by a case marking particle, each element of which has a set of co-occurring verbs in a sentence. The other is a set of verls accompanied by a case marking particle, each element of which has a set of cooccurring nouns in a sentence. We call these two co-occurrence data sets near co-occurrence data sets. Thereafter, we apply the data sets to the processing of lomonyns. 'Two strategies are used to approach the problem. The first uses the near co-occurrence data sets to select the most feasible word among homonyms in the scope of a sentence. The aim is to evaluate the possible existence of a near co-occurrence relation, or co-occurrence relation between a noun and a verb within a sentence. The second evaluates the possible existence of a far co-occurrence relation, referring to a cooccurrence relation among words in different sentences. This is achieved by constructing far cooccurrence data sets from near co-occurrence data sets in the course of processing input sentences.

\section{Co-occurrence data sets}

The near co-occurrence data sets are defined.

The first near co-occurrence data set is the set $\Sigma_{N_{\text {near }}}$, each element of which $(n)$ is a triplet consisting of a noun, a case marking particle, and a set of verbs which co-occur with that noun and particle pair in a sentence, as follows:

$$
n=\left(\text { noun, particle, }\left\{\left(v_{1}, k_{1}\right),\left(v_{2}, k_{2}\right), \cdots\right\}\right)
$$

In this description, particle is a Japanese case marking particle, such as が (nominative case), t (accusative case), or $に$ (dative case), $v_{i}(i=$ $1,2, \cdots)$ is a verb, and $k_{i}(i=1,2, \cdots)$ is the frequency of occurrence of the combination noun, particle and $v_{i}$, which is determined in the course of constructing $\Sigma_{N_{n e a r}}$ from corpora. The following are examples of the elements of $\Sigma_{N_{\text {near }}}$.

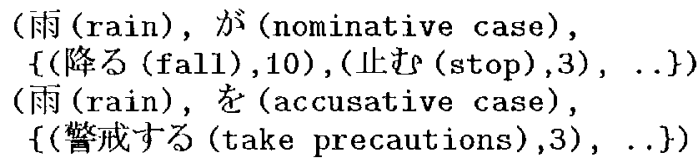


The second near co-occurrence data set is the set $\Sigma_{V_{\text {near }}}$, each element of which $(v)$ is a triplet consisting of a verb, a case marking particle, and a set of nouns which coroccur with that verb and particle pair in a sentence, as follows:

$$
v=\left(\text { verb, particle },\left\{\left(n_{1}, l_{1}\right),\left(n_{2}, l_{2}\right), \cdots\right\}\right)
$$

In this description, particle is a Japanese case marking particle, $n_{i}(i=1,2, \cdots)$ is a noun, and $l_{i}(i=1,2, \cdots)$ is the frequency of occurrence of the combination verb, particle and $n_{i}$. The following are examples of the elements of $\Sigma_{V_{n e a r},} . \Sigma_{V_{n e a n} \text {. }}$ can be constructed from $\Sigma_{N_{n e u}}$, and vice versa.

(降る (fall)，が (nominative case)，

$\{($ 雨 (rain), 10$)$, (雪 (snow), 8$), \ldots\}$ )

(降る (fall), に (dative case),

$\{($ 九州 (Kyushu), 1$) ， .$.$\} )$

\section{Processing homonyms in a simple sentence}

Using the near co-occurrence data sets, the most feasible word among possible homonyms can be selected within the scope of a sentence. Our hypothesis states that the most feasible noun or combination of nouns has the largest number of verbs with which it can co-occur in a sentence.

The structure of an input Japanese sentence written in kana-characters can be simplified as follows:

$$
N_{1} \cdot P_{1}, N_{2} \cdot P_{2}, \cdots, N_{m} \cdot P_{m}, V
$$

where $N_{i}(i=1,2, \cdots, m)$ is a noun, $P_{i}(i=$ $1,2, \cdots, m)$ is a particle and $V$ is a verl).

\subsection{Procedure}

Following is the procedure for finding the most feasible combination of words for an input kana string which has the above simplified Japanese sentence structure. This procedure can also accept an input kana-string which does not include. a final position verb.

Step1 Let $m=0$ and $T_{i}=\varepsilon(i=1,2, \cdots)$.

Step2 If an input kana-string is null, go to Step4. Otherwise read one block of kana-string, that is $N \cdot P$ or $V$, from the left side of the input kana-string. And delete the one block of kana-string from the left side of the input kana-string.

Step3 Find all homonymic kanji-variants $W_{k}(k=1,2, \cdots)$ for the kana-string $N$ or $V$ which is read in Step2.

Increase $m$ by 1 .

For each $W_{k}(k=1,2, \cdots)$ :

1. If $W_{k}$ is a noun, retrieve $\left(W_{k}, P, V_{k}\right)$ from the near co-occurrence data set $\Sigma_{N_{n e a r}}$ and add the doublet $\left(W_{k}, V_{k}\right)$ to $T_{m}$.

2. If $W_{k}$ is a verb, add the doublet $\left(W_{k},\left\{\left(W_{k}, 0\right)\right\}\right)$ to $T_{m}$.
Go to Step2.

Step4 From $T_{i}(i=1,2, \cdots, m)$, find the combination:

$$
\begin{gathered}
\left(W_{1}, V_{1}\right)\left(W_{2}, V_{2}\right), \cdots,\left(W_{m}, V_{m}\right) \\
\left(W_{i}, V_{i}\right) \in T_{i}(i=1,2, \cdots, m)
\end{gathered}
$$

which has the largest value of $\left|\cap\left(V_{1}, V_{2}, \cdots, V_{m}\right)\right|$. Where the function $\bigcap\left(V_{1}, V_{2}, \cdots, V_{m}\right)$ is defined as follows.

$$
\begin{gathered}
\bigcap\left(V_{1}, V_{2}, \cdots, V_{m}\right)=\left\{\left(v, \sum_{i=1}^{m} k_{i}\right) \mid\right. \\
\left.\left(v, k_{1}\right) \in V_{1} \wedge \cdots \wedge\left(v, k_{m}\right) \in V_{m}\right\} \\
\text { And }\left|\cap\left(V_{1}, V_{2}, \cdots, V_{m}\right)\right| \text { is defined: } \\
\left|\bigcap\left(V_{1}, V_{2}, \cdots, V_{m}\right)\right|=\sum_{(v, k) \in \bigcap\left(V_{1}, V_{2}, \cdots, V_{m}\right)}^{k}
\end{gathered}
$$

The sequence of words $W_{1}, W_{2}, \cdots, W_{m}$ is the most feasible combination of homonymic kanji-variants for the input kana-string.

\subsection{An example of processing homonyms} in a simple sentence

Following is an example of homonym processing using the above procedures.

For the input kana-string

$$
\text { “かわに恬し孝 (kawa ni hashio)" }
$$

“加 (kawa)" means a river and “忙し (hashi)" means a bridge. “加 (kawa)" and "住し (hashi)" both have homonymic kanji-variants:

$$
\begin{array}{ll}
\text { homonyms of "加わ(kawa)": } & \text { 川 (river) } \\
& \text { 皮 (leather) } \\
\text { homonyms of “はし (hashi)"： } & \text { 橋 (bridge) } \\
& \text { 箬 (chopsticks) }
\end{array}
$$

The near co-occurrence data for "川 (river)" and “皮(leather)" followed by the particle “に (dative case)" and the near co-occurrence data for "橋 (bridge)" and "簀 (chopsticks)" followed by the particle “学 (accusative case)" are shown below.

(川 (river), に, \{( 行く (go),8), (架忛る (build) , 6)， (落与 (drop), 5)\})

（攵 (leather)，に，\{(塗る (paint), 6$) ，$ (触る (touch) , 3)\})

(橋 (bridge)，灰，\{(渡る (walk across), 9)， (架ける (build) ,7)， (落す (drop) , 4)\})

(箸 (chopsticks)，を,\{(使う (use),7)， (落寸 (drop), 3)\})

Following the procedure, the resultant frequency values are as follows:

$$
\begin{array}{ll}
\text { 川に橋を } & 22\{\text { 架ける, 落す }\} \\
\text { 川に䈐を } & 8\{\text { 落す }\} \\
\text { 皮に橋を } & 0\{\} \\
\text { 皮に箸を } & 0\{\}
\end{array}
$$

Therefore, the nost feasible combination of words is "川 (river) に橋 (bridge) を." 


\section{An experiment on processing homonyms in a simple sentence}

\subsection{Preparing a dictionary and a co-occurrence data file}

\subsection{1 a noun file}

A noun file including 323 nouns, which consists of 190 nouns extracted from text concerning current topics and their 133 homonyns, was prepared.

\subsection{2 a co-occurrence data file}

A co-occurrence datia file was prepared. The record format of the file is specified as follows:

[nomn, case marking particle, verb, the frequency of occurrence]

where case marking particle is chosen from 8 kinds of particles, namely, “が”,“を”, “に”,“へ”,“と”,“か ら”,"より”,“で”.

It includes 25,665 records of co-occurrence relation (79 records per noum) for the nouns in the noun file by merging 11,294 records from $\mathrm{EDR}$ Co-occurrence Dictionary(EDR,1994) with 15,856 records from handmade simple sentences.

\subsection{3 an input file and an answer file}

An input file for an experiment, which inclucles 1,129 simple sentences written in kana alphabet, and an answer file, which includes the sane 1,129 sentences written in kanji characters, were prepared. Tere, every nom of the sentences in the files was chosen from the nown file.

\subsection{4 a word dictionary}

A word dictionary, which consists of 323 11011ns in the noun file and 23,912 verbs in a Japanese? dictionary for kana-to-kanji conversion ', was prepared. It is used to find all homonymic kamjit variants for each noun or verb of the sentences in the input, file.

\subsection{Hxperiment results}

An experiment on processing homony nus in a sinple sentence was carried out. In this experiment, kana-to-kanji conversion was applied to each of the sentences, or the input kana-strings, in the above input file and the near co-oceurnence data sets were constructed from the above cooccurrence data file. Table1 shows the results of kana-to-kanji conversion in the following two cases. In the first case, an input kana-string does not inclucle a final position verb. It means that each verb of the kanastrings in the input file is neglected. In the second case, an input banastring includes a final position verb. The experiment has shown that the conversion is carried ont at the accuracy rate of $79.6 \%$ per word, where the conversion rate is $93.1 \%$ per word, in the first

\footnotetext{
${ }^{1}$ This dictionary was made by $\Lambda I$ Soft $C o$.
}

casce. In the sane way, the accuracy rate is $93.8 \%$ per word, where the conversion rate is $14.5 \% \mathrm{per}$ word, in the second case. And then, we also conlucted the same experiment by using the method based on the word occurrence frequency to conpare onr method with an ordinary method. It has show that the accuracy rate is $72.2 \%$ per word in the first case, and $77.8 \%$ per word in the second case. Wo can find the accuracy rate by our wethod is $7.4 \%$ higher in the first case and $16.9 \%$ ligher in the second case compared with the ordinary method. It is clarified that our method is more effective than the ordinary method based on the word oceurrence frequency.

\section{An approximation of the far co-occurrence relation}

We can approximate the far co-occurrence relation, which is co-occurrence relation among words in a sequence of sentences, from near cooccurrence data scts. The far co-occurrence data sets are described as follows:

$$
\begin{aligned}
& \Sigma_{N_{f u},}=\left\{\left(n_{1}, t_{1}\right),\left(n_{2}, t_{2}\right), \cdots,\left(n_{l_{n}}, t_{l_{n}}\right)\right\} \\
& \Sigma_{V_{f, u}}=\left\{\left(u_{1}, u_{1}\right),\left(v_{2}, u_{2}\right), \cdots,\left(v_{l_{v}}, u_{l_{v}}\right)\right\}
\end{aligned}
$$

where $n_{i}\left(i=1,2, \cdots, l_{n}\right)$ is a noun, $t_{i}$ is the priority value of $n_{i}, v_{i}\left(i=1,2, \cdots, l_{v}\right)$ is a verb and $u_{i}$ is the priority value of $v_{i}$.

The procedure for producing the far cooceurvence data sets is:

Step1 Clear the far co-occurrence data sets.

$$
\begin{aligned}
& \Sigma_{N_{f u r}}=\epsilon \\
& \Sigma_{V_{f a r}}=\epsilon
\end{aligned}
$$

Step2 After cach fixing of nomn $N$ among homo nyms in the process of kana-to-kaniji conversion, renew the far co-occurrence data sets $\Sigma_{N_{f i b},}$ and $\Sigma_{V_{f}, r}$ by following these steps:

1. Change all priority values of $t_{i}(i=$ $\left.1,2, \cdots, l_{n}\right)$ in the set $\Sigma_{N_{f a},}$ to $f\left(t_{i}\right)$ (for example, $\left.f\left(t_{i}\right)=0.95 t_{i}\right)$. This process is intended to decrease priority with the

\begin{tabular}{|c|c|c|}
\hline & $\begin{array}{l}\text { For sentences } \\
\text { withont a verts }\end{array}$ & $\begin{array}{l}\text { For sentences } \\
\text { with a verb }\end{array}$ \\
\hline $\begin{array}{l}\text { Conversion rate } \\
\text { per sentence }\end{array}$ & $\begin{array}{c}1053 / 1129 \\
(93.3 \%)\end{array}$ & $\begin{array}{c}166 / 1129 \\
(14.7 \%)\end{array}$ \\
\hline $\begin{array}{l}\text { Acruacy rate } \\
\text { per sentence }\end{array}$ & $\begin{array}{c}66371053 \\
(63.0 \%)\end{array}$ & $\begin{array}{l}136 / 166 \\
(81.9 \%)\end{array}$ \\
\hline $\begin{array}{c}\text { Conversion rate } \\
\text { per word }\end{array}$ & $\begin{array}{c}2155 / 2315 \\
(93.1 \%)\end{array}$ & $\begin{array}{c}500 / 3444 \\
(14.5 \%)\end{array}$ \\
\hline $\begin{array}{c}\text { Accuracy rate } \\
\text { per word }\end{array}$ & $\begin{array}{c}1716 / 2155 \\
(79.6 \%)\end{array}$ & $\begin{array}{l}469 / 500 \\
(93.8 \%)\end{array}$ \\
\hline
\end{tabular}
passage of time.

Table 1: Experinent results on processing homonyms in a simple sentence 
2. Change all priority values of $u_{i}(i=$ $\left.l_{,}, 2, \cdots, \cdots, l_{v}\right)$ in the set $\Sigma_{V_{J a r}}$ to $f\left(u_{i}\right)$ as well.

3. Let $N$ be the noun determined in the process of kana-to-kanji conversion. Find all

$$
\left(v_{i}, k_{i}\right)(i=1,2, \cdots, q)
$$

which co-occur with the noun $N$ followed by any particle, in the near co-occurrence data set $\Sigma_{N_{n e a r}}$. Add new elements

$$
\left(v_{i}, g\left(k_{i}\right)\right)(i=1,2, \cdots, q)
$$

to the set $\Sigma_{V_{f a r}}$. If an element with the same verb $v_{i}$ already exists in $\Sigma_{V_{f a r}}$, add the value $g\left(k_{i}\right)$ to the priority value of that element instead of the new element. Here, $g\left(k_{i}\right)$ is a function for converting frequency of occurrence to priority value. For example,

$$
g\left(k_{i}\right)=1-\left(1 / k_{i}\right)
$$

4. Let $v_{i}$ be the verb described in the previous step. Find all

$$
\left(n_{j}, l_{j}\right)(j=1,2, \cdots, q)
$$

which co-occur with the verb $v_{i}$ and any particle in the near co-occurrence data set $\Sigma_{V_{\text {near }}}$. Add new elements

$$
\left(n_{j}, h\left(k_{i}, l_{j}\right)\right)(j=1,2, \cdots, q)
$$

to the set $\Sigma_{N_{f a r}}$. If an element with the same noun $n_{j}$ already exists in $\Sigma_{N_{f a r}}$, add the value $h\left(k_{i}, l_{j}\right)$ to the priority value of that element instead of the new element. Here, $h\left(k_{i}, l_{j}\right)$ is a function for converting frequency of occurrence to priority value. For example,

$$
h\left(k_{i}, l_{j}\right)=g\left(k_{i}\right)\left(1-\left(1 / l_{j}\right)\right)
$$

\section{Processing homonyms in a sequence of sentences}

Using the far co-occurrence data sets defined in the previous section, the most feasible word among homonyms can be selected in the scope of a sequence of sentences according to the following two cases.

Case1 An input word written in kana-characters is a noun.

Case2 An input word written in kana-characters is a verb.

\subsection{Procedure for case1}

Step1 Find set $S_{n}$ :

$$
S_{n}=\left\{\left(N_{1}, T_{1}\right),\left(N_{2}, T_{2}\right), \cdots\right\}
$$

where $N_{i}(i=1,2, \cdots)$ is a homonymic kanjivariant for the input word written in kanacharacters and $T_{i}$ is the priority value for homonym $N_{i}$, which can be retrieved from the far co-occurrence data set $\Sigma_{N_{J a r}}$.
Step2 The noun $N_{i}$ which has the greatest $T_{i}$ priority value in $S_{n}$ is the most feasible noun for the input word written in kana-characters.

\subsection{Procedure for case2}

Step1 Find set $S_{v}$ :

$$
S_{v}=\left\{\left(V_{1}, U_{1}\right),\left(V_{2}, U_{2}\right), \cdots\right\}
$$

Here, $V_{j}(j=1,2, \cdots)$ is a homonymic kanjivariant for the input word written in kanacharacters and $U_{j}$ is the priority value for homonym $V_{j}$, which can be retrieved from the far co-occurrence data set $\Sigma_{V_{f a r}}$.

Step2 The verb $V_{j}$ which has the greatest $U_{j}$ priority value in $S_{v}$ is the most feasible verb for the input word written in kana-characters.

\section{Conclusion}

We have proposed two new methods for processing Japanese homonyms based on the co-occurrence relation between a noun and a verb in a sentence which can be obtained easily from corpora. Using these methods, we can evaluate the co-occurrence relation of words in a simple sentence by using the near co-occurrence data sets obtained from corpora. We can also evaluate the co-occurrence relation of words in different sentences by using the far co-occurrence data sets constructed from the near co-occurrence data sets in the course of processing input sentences. The far co-occurrence data sets are based on the proposition that it is more practical to maintain a relatively small amount of data on the semantic relations between words, being changed dynamically in the course of processing, than to maintain a luge universal "thesaurus" data base, which does not appear to have been built successfully.

An experiment of kana-to-kanji conversion by the first method for 1,129 input simple sentences has shown that the conversion is carried out in $93.1 \%$ per word and the accuracy rate is $79.6 \%$ per word. It is clarified that the first method is more effective than the ordinary method based on the word occurrence frequency.

In the next stage of our study, we intend to evaluate the second method based on the far cooccurrence data sets by conducting experiments.

\section{References}

Kobayashi, T., et al. 1992. Realization of Kana-to-Kanji Conversion Using Neural Networks. Toshiba Review, Vol.47, No.11, pages 868870, Japan.

Yamamoto, K., et al. 1992. Kana-to-Kanji Conversion Using Co-occurrence Groups. Proc. of 44th Conference of IPSJ, 4p-11, pages 189-190, Japan.

EDR. 1994. Co-occurrence Dictionary Ver.2, TR-043, Japan. 